\title{
The sticky truth about angiogenesis and thrombospondins
}

\author{
Judith A. Varner \\ Moores UCSD Cancer Center, La Jolla, California, USA.
}

\begin{abstract}
The formation of new blood vessels, a process known as angiogenesis, is important for embryonic development and wound healing as well as the development of cancer and inflammation; therefore, angiogenesis is a valuable target for clinical intervention. Both logic and empiricism suggest that a balance of stimulatory and inhibitory switches is required for orderly formation of blood vessels. Thrombospondins 1 and 2 were among the first natural angiogenesis inhibitors to be identified. However, the cellular origins and mechanisms of action of these important proteins during angiogenesis have remained largely unknown. Studies by Kopp et al., presented in this issue of the JCI, clarify some of these issues by revealing that megakaryocytes and their "sticky" wound-healing progeny, platelets, are important sources of thrombospondins 1 and 2 and that these thrombopoietic cells play key roles in controlling blood vessel formation during hematopoiesis and ischemic wound healing (see the related article beginning on page 3277 ).
\end{abstract}

Blood vessels convey the 3 essential elements of life (food, water, and air, or oxygen) to all tissues. The development of these conduits of life-giving essentials allowed the evolution of large organisms with complex tissues but required precise control mechanisms - mechanisms that are often perturbed during disease progression.

Identification of the systems controlling the formation of blood vessels has been the subject of over 30 years of intensive research. Studies of normal angiogenesis, such as occurs during wound healing, suggest that it is a finely regulated, short-term process that is initiated by the release of stimulatory factors from platelets, "sticky" circulating cells that plug wounds in blood vessel walls or stromal cells, such as fibroblasts. New vessel formation is then inhibited by the absence of stimulatory factors and/or by naturally arising inhibitory factors that may include proteolytic fragments of extracellular matrix proteins. Early developments in the field of angiogenesis research led to the discovery of many angiogenesis-stimulating factors, including the well-known VEGF, and to the discovery of naturally occurring angiostatic agents,

Nonstandard abbreviations used: SDF-1, stromal cell-derived factor 1; TSP, thrombospondin; TSP-DKO, TSP double-knockout (mice).

Conflict of interest: The author has declared that no conflict of interest exists.

Citation for this article: J. Clin. Invest. 116:3111-3113 (2006). doi:10.1172/JCI30685. such as the thrombospondins (TSPs) (1-3). TSP1 was the first naturally arising angiogenesis inhibitor to be described (1-3). Shortly thereafter, a second TSP, TSP2, was also found to inhibit angiogenesis (4). Although much is known about these natural angiostatic proteins, much remains uncertain. For example, it has not been clear which cells express TSP1 and TSP2 during angiogenesis in vivo. The exact mechanisms by which TSPs inhibit angiogenesis in vivo have also remained elusive. The studies by Kopp et al. in this issue of the JCI shed new light upon these mysteries (5).

\section{TSP1 and TSP2}

TSPs are a family of 5 structurally related extracellular matrix proteins with the capacity to bind both cell surface receptors and other extracellular matrix proteins (6). TSP1 and TSP2 are very similar structurally and functionally; both have been implicated as inhibitors of angiogenesis, endothelial cell survival, and endothelial cell migration (7). TSP1, the first of the family to be described, is a thrombin-sensitive extracellular matrix protein that is released from platelet granules after activation (8). Importantly, it is the most abundant protein in platelet $\alpha$-granules. It was isolated from platelets and was localized to megakaryocytes and the extracellular matrix as early as $1978(8,9)$. It is also expressed by endothelial cells and fibroblasts in vitro and by certain tumor cells in vitro and in vivo $(10,11)$. A similar protein, TSP2, was identified in endothelial cells a few years later (4). TSP1 is an arginine-glycine-aspartic acid-containing ligand for integrin $\alpha v \beta 3$ (6). It is also a ligand for CD36, a G protein-coupled receptor on endothelial cells (6). Several studies indicate that TSP1 binding to CD36 inhibits endothelial cell migration and induces endothelial cell apoptosis in vitro (12-15). TSP1 also binds to other ECM components, such as fibrinogen, fibronectin, and proteoglycans, and plays a role in platelet aggregation (16).

In 1990, TSP1 was first described as an inhibitor of angiogenesis, as it blocked the formation of new blood vessels in the cornea in vivo in response to basic FGF $(1,3)$ and blocked endothelial cell tube formation and cell migration in vitro (2). Additional studies showed that TSP1 could serve as a naturally occurring tumor suppressor by inhibiting tumor angiogenesis (3). Recent studies indicate that loss of TSP1 or TSP2 during embryonic development is not lethal but results in increased vascular density $(17,18)$. Various studies indicate that adult TSP2 $2^{-/-}$and $T S P 1^{-/-}$mice exhibit increased vascular density during wound healing, retinal development, and tumor growth $(17,18)$. In contrast, TSP1 transgenic mice exhibit decreased vascular density (19).

\section{What is the source of thrombospondins?}

The tissue source of TSP during angiogenesis has been unclear. During embryonic development, TSP2, but not TSP1, is expressed in endothelia and developing connective tissues (20). In contrast, TSP1, but not TSP2, is expressed in megakaryocytes and in the developing kidney and gut (20). Other studies have shown that TSP1 is expressed by megakaryocytes and platelets in the adult animal $(8,9)$ and that both TSPs are expressed in endothelial cells cultured in vitro $(2,10,11)$. Fibroblasts also can express TSP1 and TSP2 (1, 21). As endothelial cells, fibroblasts, and platelets are all present in healing wounds and tumors, it has not been clear which cell 

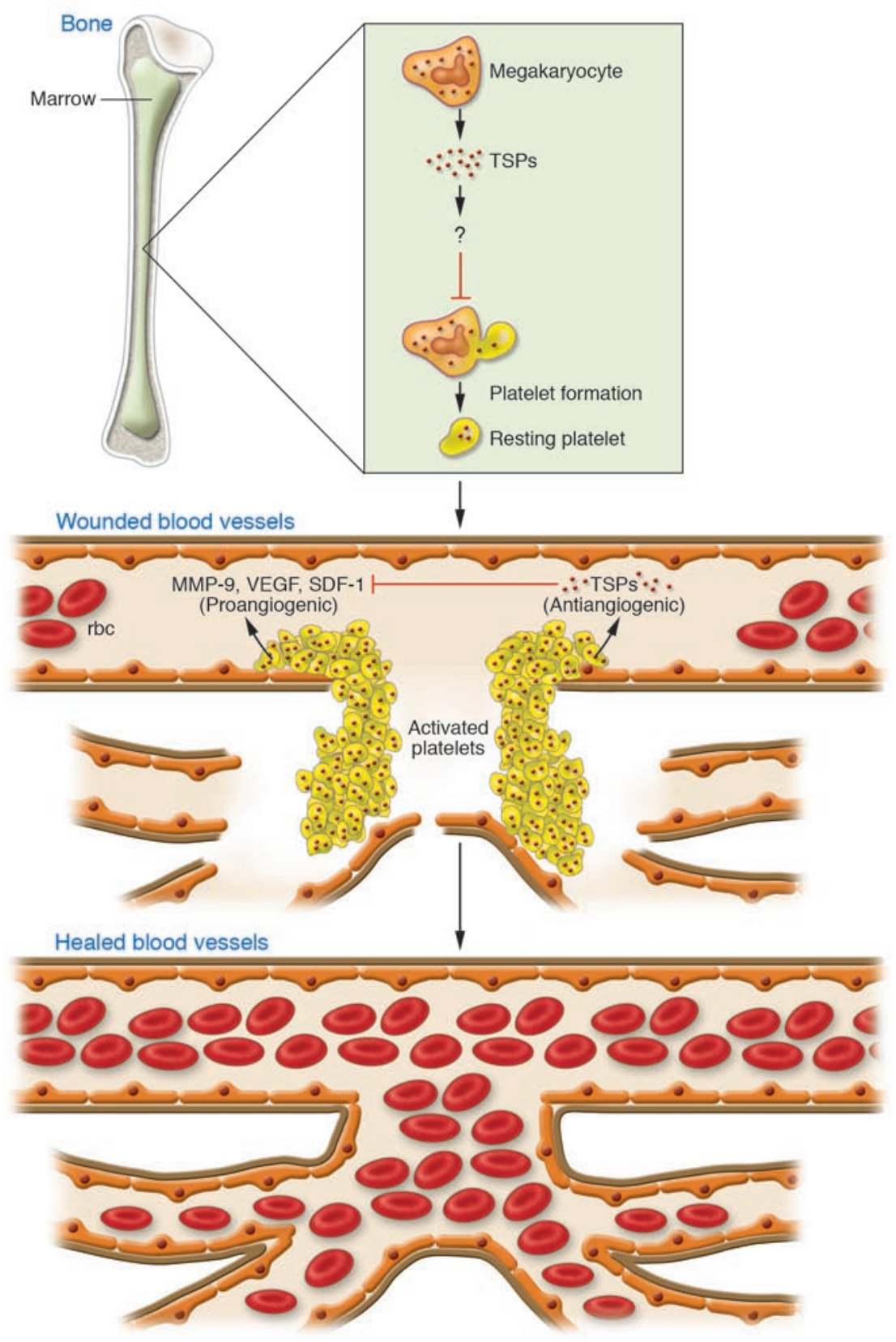

type is responsible for the secretion of TSPs during angiogenesis inhibition.

As platelets release TSP1 upon activation, they may control a key angiogenic switch. Indeed, the studies in this issue of the JCI by Kopp et al. (5) elegantly show that thrombopoietic cells regulate an angiogenic switch by secreting the inhibitory TSPs (Figure 1). These studies suggest that angiogenesis is regulated by the absolute numbers of megakaryocytes in bone marrow or platelets in ischemic tissue and by the amount of TSP1 and TSP2 within these cells. These studies show that within the bone marrow, megakaryocytes, rather than endothelial cells or other cells, express TSPs.

Key studies in this article show that the recovery of blood platelet production (thrombopoiesis) after suppression of bone marrow activity (myelosuppression) is enhanced in mice that are deficient in both TSP1 and TSP2 (5). After myelosuppression, megakaryocyte and platelet levels in TSP double-knockout (TSP-DKO) mice more rapidly returned to normal compared with those in wild-type mice, apparently as a result of accelerated revascularization of myelosuppressed bone marrow. Importantly, Kopp et al. also show that wild-type

\section{Figure 1}

TSPs released by thrombopoietic cells during wound healing act as angiogenic switches and control the extent of revascularization. Megakaryocytes in the bone marrow give rise to platelets that carry large stores of TSPs in their $\alpha$-granules. Circulating platelets traffic from bone marrow to sites of injury and, for example, adhere to components of the blood vessel wall in order to plug gaps in wounded blood vessels. Simultaneously these platelets release pro-angiogenic growth factors, such as VEGF, MMP-9, and SDF-1. In this issue of the $\mathrm{JCl}$, Kopp et al. (5) show that TSPs released by megakaryocytes and platelets have an antiangiogenic effect. The TSPs act as an angiogenic "switch," binding to MMP-9 and SDF-1 to inhibit the angiogenic cascade and limit the extent of vascularization. The signals that control the balance and timing of the release of pro- and antiangiogenic factors in tissues have yet to be fully determined. The authors also show that TSP1 and TSP2 negatively regulate megakaryocyte proliferation in the bone marrow and consequently regulate platelet numbers in the blood.

megakaryocytes embedded in Matrigel stimulate angiogenesis; however, megakaryocytes from TSP2 $2^{-/-}$and TSP1 $1^{-/-}$DKO mice stimulate angiogenesis to a much greater extent than do wild-type megakaryocytes. The important role of megakaryocytes and platelets in regulating angiogenesis is particularly well demonstrated by studies in which bone marrow from TSP-DKO mice was transplanted into wild-type mice. In these studies, wild-type mice transplanted with bone marrow from TSP-DKO mice exhibited enhanced experimental hind limb ischemic angiogenesis whereas TSPDKO mice transplanted with bone marrow from wild-type mice did not. These studies also show that wild-type platelets adoptively transferred into TSP-DKO mice deposited TSP near blood vessels. As platelets are frequently found at sites of healing wounds and within tumors $(22,23)$, these studies highlight the important role of platelets in regulating angiogenesis.

Importantly, the studies presented in this paper (5) indicate that by regulating vascular density in the bone marrow, TSP1 and TSP2 also control megakaryocyte proliferation in the bone marrow and thereby platelet numbers in the peripheral blood. This article further delineates a mechanism whereby platelet-derived TSP inhibits angiogenesis: TSP-DKO mice exhibit increased MMP-9 activity and enhanced release of stromal cell-derived factor 1 (SDF-1) from platelets. As MMP-9 can 
release proangiogenic factors such as SDF-1 from matrix-bound stores (24), these results suggest that TSP1 and TSP2 inhibit angiogenesis by promoting the sequestration of key proangiogenic factors. These studies suggest that TSPs inhibit angiogenesis by acting as sponges that absorb angiogenesis-stimulating molecules. Therefore, thrombopoietic cell TSP is well established by these novel findings as an angiogenesissuppressing switch (Figure 1).

\section{Remaining questions about the roles of TSPs in angiogenesis}

Although a number of important questions about the roles of TSPs and platelets in angiogenesis have been answered by these studies (5), other questions remain unanswered. Platelets have long been thought to play a role in the initiation of angiogenesis during wound healing by secreting factors such as VEGF and SDF-1. If platelets also play a key role in angiogenesis suppression, under what circumstances do platelets stimulate rather than inhibit angiogenesis? Is there a temporal difference in the release of pro- and antiangiogenic molecules from platelets? Fibroblasts, endothelial cells, and other cells also express TSPs. Under what circumstances do these pools of TSP control angiogenesis? What are the signals that control the net balance of pro- and antiangiogenic factors released in a tissue? The body of evidence that TSPs 1 and 2 can inhibit angiogenesis suggests that these proteins may be useful clinically. In fact, a phase I clinical trial evaluating the safety and efficacy of a TSP peptidomimetic, ABT-510, in patients with solid tumors was recently completed with no toxicities reported (25). With the publication of these studies, it is clear that the study of TSPs will continue to provoke new insights and suggest new paradigms of angiogenic fine-tuning.

Address correspondence to: Judith A. Varner, Moores UCSD Cancer Center, 3855 Health Sciences Drive, La Jolla, California 920930819, USA. Phone: (858) 822-0086; Fax: (858) 822-1325; E-mail: jvarner@ucsd.edu.

1. Good, D.J., et al. 1990. A tumor suppressor-dependent inhibitor of angiogenesis is immunologically and functionally indistinguishable from a fragment of thrombospondin. Proc. Natl. Acad. Sci. U. S. A. 87:6624-6628.

2. Iruela-Arispe, M.L., Bornstein, P., and Sage, H. 1991. Thrombospondin exerts an antiangiogenic effect on cord formation by endothelial cells in vitro. Proc. Natl. Acad. Sci. U. S. A. 88:5026-5030.

3. Dameron, K.M., Volpert, O.V., Tainsky, M.A., and Bouck, N. 1994. Control of angiogenesis in fibroblasts by $\mathrm{p} 53$ regulation of thrombospondin-1. Science. 265:1582-1584.

4. Volpert, O.V., et al. 1995. Inhibition of angiogenesis by thrombospondin-2. Biochem. Biophys. Res. Commun. 217:326-332.

5. Kopp, H.-G., et al. 2006. Thrombospondins deployed by thrombopoietic cells determine angiogenic switch and extent of revascularization. J. Clin. Invest. 116:3277-3291. doi:10.1172/JCI29314.

6. Lawler, J., Weinstein, R., and Hynes, R.O. 1988. Cell attachment to thrombospondin: the role of ARG-GLY-ASP, calcium, and integrin receptors. J. Cell Biol. 107:2351-2361.

7. Armstrong, L.C., and Bornstein, P. 2003. Thrombospondins 1 and 2 function as inhibitors of angiogenesis. Matrix Biol. 22:63-71.

8. Lawler, J.W., Slayter, H.S., and Coligan, J.E. 1978. Isolation and characterization of a high molecular weight glycoprotein from human blood platelets. J. Biol. Chem. 253:8609-8616.

9. McLaren, K.M. 1983. Immunohistochemical localisation of thrombospondin in human megakaryocytes and platelets. J. Clin. Pathol. 36:197-199.

10. Mosher, D.F., Doyle, M.J., and Jaffe, E.A. 1982. Synthesis and secretion of thrombospondin by cultured human endothelial cells. J. Cell Biol. 93:343-348.

11. Jaffe, E.A., et al. 1983. Cultured human fibroblasts synthesize and secrete thrombospondin and incorporate it into extracellular matrix. Proc. Natl. Acad. Sci. U. S. A. 80:998-1002.

12. Taraboletti, G., Roberts, D., Liotta, L.A., and Giavazzi, R. 1990. Platelet thrombospondin modulates endothelial cell adhesion, motility, and growth: a potential angiogenesis regulatory factor. J. Cell Biol. 111:765-772.

13. Primo, L., et al. 2005. Identification of CD36 molecular features required for its in vitro angiostatic activity. FASEB J. 19:1713-1715.

14. Jimenez, B., et al. 2000. Signals leading to apoptosis-dependent inhibition of neovascularization by thrombospondin-1. Nat. Med. 6:41-48.

15. Yang, M., et al. 2003. Thrombospondin-1 inhibits in vitro megakaryocytopoiesis via CD36. Thromb. Res. 109:47-54.

16. Dixit, V.M., et al. 1985. A monoclonal antibody against human thrombospondin inhibits platelet aggregation. Proc. Natl. Acad. Sci. U. S. A. 82:3472-3476.

17. Kyriakides, T.R., et al. 1998. Mice that lack thrombospondin 2 display connective tissue abnormalities that are associated with disordered collagen fibrillogenesis, an increased vascular density, and a bleeding diathesis. J. Cell Biol. 140:419-430.

18. Wang, S., Wu, Z., Sorenson, C.M., Lawler, J., and Sheibani, N. 2003. Thrombospondin-1-deficient mice exhibit increased vascular density during retinal vascular development and are less sensitive to hyperoxia-mediated vessel obliteration. Dev. Dyn. 228:630-642.

19. Streit, M., et al. 2000. Thrombospondin-1 suppresses wound healing and granulation tissue formation in the skin of transgenic mice. $E M B O J$. 19:3272-3282.

20. Iruela-Arispe, M.L., Liska, D.J., Sage, E.H., and Bornstein, P. 1993. Differential expression of thrombospondin 1,2, and 3 during murine development. Dev. Dyn. 197:40-56.

21. Laherty, C.D., et al. 1992. Characterization of mouse thrombospondin 2 sequence and expression during cell growth and development. J. Biol. Chem. 267:3274-3281.

22. Weis, S., et al. 2004. Src blockade stabilizes a Flk/ cadherin complex, reducing edema and tissue injury following myocardial infarction. J. Clin. Invest. 113:885-894. doi:10.1172/JCI200420702.

23. Weis, S., Cui, J., Barnes, L., and Cheresh, D. 2004. Endothelial barrier disruption by VEGF-mediated Src activity potentiates tumor cell extravasation and metastasis. J. Cell Biol. 167:223-229.

24. Jin, D.K., et al. 2006. Cytokine-mediated deployment of SDF-1 induces revascularization through recruitment of CXCR4+ hemangiocytes. Nat. Med. 12:557-567.

25. Gietema, J.A., et al. 2006. A phase I study assessing the safety and pharmacokinetics of the thrombospondin-1-mimetic angiogenesis inhibitor ABT-510 with gemcitabine and cisplatin in patients with solid tumors. Ann. Oncol. 17:1320-1327. 